\title{
The diagnosis and treatment of papillary cystadenoma of the esophagus by the Endoscopy : a case report and literature review
}

\author{
Zhaomeng Zhuang ${ }^{1}$, Yiguang Zhang ${ }^{1}$, Min Xie ${ }^{1}$, and Shuo Chen ${ }^{1}$ \\ ${ }^{1}$ Wenzhou Integrated Traditional Chinese and Western Medicine Hospital
}

July 14, 2021

\begin{abstract}
Papillary cystadenoma is a epithelial benign tumor of the salivary gland, most commonly in the oral cavity, head and neck. There was no difference in the sex distribution of patients, and the age of onset was 30-50 years old. The clinical manifestations are: slow growth local mass, long course of disease, some can be as long as decades, most patients have no obvious conscious symptoms, a few patients can appear local pain or distension. However, papillary cystadenoma of the esophagus is very rare, and few cases have been reported. We report a case of papillary cystadenoma of the lower esophagus and its treatment by endoscopy. The patient had no obvious complaint of discomfort. Routine gastroscopy found submucosal protuberant lesions in the lower segment of the esophagus (Figure 1), and endoscopic ultrasonography suggested cystic and solid lesions originating from the muscular layer of the esophageal mucosa, thus mesenchymal tumor was preliminarily diagnosed (Figure 2). After the exclusion of relevant contraindications, preoperative examinations were completed and the tumor was completely removed by esophageal submucosal tumor excision under endoscopy (Figure 3). Postoperative pathology indicated the tumor is papillary cystadenoma, and immunohistochemistry indicated that: CK7 $(+), \mathrm{CK}(+)$, and basal cells of P63 (+) (Figure 4, 5). Due to the rare case, combined with the process of the diagnosis and treatment of this case, we reviewed the relevant reports on the papillary cystadenoma of the digestive tract in the past 60 years, in order to help clinicians to improve their understanding and treatments of the papillary cystadenoma.
\end{abstract}

The diagnosis and treatment of papillary cystadenoma of the esophagus by the Endoscopy : a case report and literature review

Zhuang zhaomeng, Zhang yiguang, Xie Min, Chen shuo*

Wenzhou Integrated Traditional Chinese and Western Medicine Hospital, Wenzhou, 325000

Corresponding Author*: Chen shuo, Email: zzmandlw@126.com

Abstract:

Objective Papillary cystadenoma is a epithelial benign tumor of the salivary gland, most commonly in the oral cavity, head and neck. There was no difference in the sex distribution of patients, and the age of onset was 30-50 years old. The clinical manifestations are: slow growth local mass, long course of disease, some can be as long as decades, most patients have no obvious conscious symptoms, a few patients can appear local pain or distension. However, papillary cystadenoma of the esophagus is very rare, and few cases have been reported.

Methods We report a case of papillary cystadenoma of the lower esophagus and its treatment by endoscopy. The patient had no obvious complaint of discomfort. Routine gastroscopy found submucosal protuberant lesions in the lower segment of the esophagus (Figure 1), and endoscopic ultrasonography suggested cystic and solid lesions originating from the muscular layer of the esophageal mucosa, thus mesenchymal tumor was 
preliminarily diagnosed (Figure 2). After the exclusion of relevant contraindications, preoperative examinations were completed and the tumor was completely removed by esophageal submucosal tumor excision under endoscopy (Figure 3). Postoperative pathology indicated the tumor is papillary cystadenoma, and immunohistochemistry indicated that: CK7 $(+)$, CK $(+)$, and basal cells of P63 (+) (Figure 4-6).

Results Due to the rare case, combined with the process of the diagnosis and treatment of this case, we reviewed the relevant reports on the papillary cystadenoma of the digestive tract in the past 60 years, in order to help clinicians to improve their understanding and treatments of the papillary cystadenoma.

keywords: Endoscopic submucosal mass excision - papillary cystadenoma of the esophagus

\section{declare conflict of interest statement}

no conflict of interest

\section{Case introduction:}

The 53-year-old male patient had a smoking history of 30 years, with an average of 10 cigarettes per day, and no unsatisfactory clinical symptoms. Gastroduodenoscopy was performed in Wenzhou Integrated Traditional Chinese and Western Medicine hospital for routine health examination, and tumor of the lower esophagus was found. She came to Wenzhou Integrated Traditional Chinese and Western Medicine hospital for endoscopic treatment on January 19, 2021. Endoscopic results showed that a mucosal hump was observed 42cm away from the incisor, with a diameter of about $1.3^{*} 1.2 \mathrm{~cm}$, and the mucosa on the surface was smooth (Figure 1). Endoscopic ultrasonography showed that $42 \mathrm{~cm}$ from the incisor of the esophagus, there was a low-echo mass originated from the muscularis mucosa, with uneven echo and clear boundary. The measured cross-section was $0.74 * 0.51 \mathrm{~mm}$ (Figure 2). The initial diagnosis of it was esophageal stromal tumor, and the nature of this tumor should await the results of pathological examination. After admission, relevant preoperative examinations were completed, and endoscopic resection of lower esophageal mass was performed on January 20, 2021 after surgical contraindications were eliminated (Fig 3). Postoperative pathological findings: one grayish white nodules, diameter $0.8 \mathrm{~cm}$, clear boundary, no capsule, section of grayish white, soft texture. (Fig. 4) The lesion consists of variously-sized, dilated glandular lumens surrounded by dense, proliferative lymphoid tissue. The glandular lumen is filled with abundant clastic red - stained secretions. The inner wall of the glandular lumen is lined with double-layer epithelial cells, and the inner epithelial cells are atrophied. The cells are flat, cubic, and a few are columnar. The epithelial hyperplasia in the focal area folds into the lumen and shows papillary growth. The basal cells in the outer layer of the glandular lumen were flat or cubic, with round or flat nuclei, rare cytoplasm and some transparent cytoplasm. The morphology of the two layers of cells was mild and no pathological mitosis was observed. Immunohistochemical staining (Figure 5-6) showed that The inner epithelial cells of the glandular lumen cytokeratin (Cytokeratin, CK) positive, CK7 positive, P63 positive cell proportion < 1\%, CD20 negative, CD117 negative, thyroid transcription factor-1 (TTF-1) negative, Lymphatic endothelial markers (D2-40) negative, tumor cell proliferation index in double epithelial cells (Ki-67) negative.

\section{Discussion}

Papillary cystadenoma usually occurs in salivary gland tissues such as oral cavity, head and neck, and has a certain risk of malignant transformation. Some reports have shown that this type of tumor can also be distributed in other parts of the digestive tract, such as lip, tongue, nasopharynx, orbit, zygomatic part, larynx, esophagus, gastroesophageal junction, cardia, gastric antrum, transverse colon, rectum, anus and so on. By reviewing the relevant literature, we analyzed the characteristic of gastrointestinal papillary cystadenoma. The clinical and pathological features were summarized.

Papillary cystadenoma occurring in the digestive tract is extremely rare, and can happen in any part of the digestive tract. At present, the pathogenesis is not clear. Some literatures believe that it may be related to congenital ectopic metaplasia of salivary gland ${ }^{[1]}$. The clinical manifestations are obviously related to the location of the lesions. Among which the cases occurred in the larynx, esophagus, gastroesophageal junction, cardia and other places can be treated for dysphagia, choking feeling, acid regurgitation and other symptoms 
${ }^{[2-4]}$; When the cases occurred in the transverse colon, rectum, anus and other lower digestive tract can be treated for bleeding, abdominal pain, anal foreign body sensation and other symptoms ${ }^{[5-8]}$; Some cases such as the tumor was in the gastric antrum may have no obvious clinical symptoms ${ }^{[9]}$. Endoscopic presentation of the disease are submucosal protuberant lesions or polypoid protuberant lesions. Endoscopic ultrasonography may showed hypoechoic lesions confined to the mucosa or submucosa, with diameters ranging from 1 to 2 $\mathrm{cm}$. Clinically, it needs to be differentiated from gastrointestinal stromal tumor, leiomyoma, neuroendocrine tumor, cyst, lipoma, heterotopic pancreas and other diseases ${ }^{[10]}$.

The pathological features were ${ }^{[11-13]}$ : the tumor was round in shape, with the largest diameter of $1-3 \mathrm{~cm}$, nodular on the surface and clear boundary with surrounding tissues. The section is white or grayish white, solid or with different sizes of cysts. There are mucoid substances in the cysts, and small papillae can be seen in the larger cysts. Microscopic examination showed that the tumor was composed of mucous cells and cuboidal cells. The two kinds of cells are staggered. The mucus cells were columnar or cuboidal, with round nucleus, located at one end of the cells. They were small in size, stained deeply, and stained lightly in cytoplasm. The cytoplasm of cuboidal cells is eosinophilic, the boundary is not clear, the nucleus is round or oval, the volume is large, and the staining is light. In papillary cystadenoma, the glandular cavity is extremely enlarged into a cystic cavity, which is lined by columnar or cuboidal glandular epithelial cells, and many proliferating papillae protrude into the cavity. The epithelial cells of the nipple are high columnar, with secretion. There are connective tissue fiber bundles in the center of the nipple, without lymphoid tissue. There are eosinophils in the capsule.

Currently, the understanding of the nature of papillary cystadenoma is not consistent. Most researchers consider it to be benign tumor but locally invasive. Some cases showed severe atypical hyperplasia, or highly differentiated papillary carcinoma and papillary cystadenocarcinoma due to abundant papillary proliferation of ducts. Or abominable mucoepidermoid carcinoma ${ }^{[14-17]}$. Therefore, the disease has a certain risk of malignant transformation, which should be paid attention to by clinicians.

At present, papillary cystadenoma should be treated with surgery. Endoscopic submucosal dissection and endoscopic submucosal mass removal (ESD, ESE) is recommended for papillary cystadenomas of the digestive tract. Patients were sent to regular endoscopic follow-up after diagnosis by pathology and immunohistochemistry after surgery. The were no complications or recurrence case reported after the endoscopic resection or surgical resection in benign cases.

Therefore, based on this case and a review of the relevant literature, we concluded that papillary cystadenoma can occur in any part of the digestive tract and lacks specific clinical and endoscopic manifestations. And the diagnosis of papillary cystadenoma should be confirmed by pathological examination after complete resection of the lesion by endoscopic surgery or surgical resection. Although the incidence of papillary cystadenoma of the digestive tract is extremely low, it has a certain risk of malignant transformation, so it needs to be paid more attentions to by endoscopologists and pathologists. 

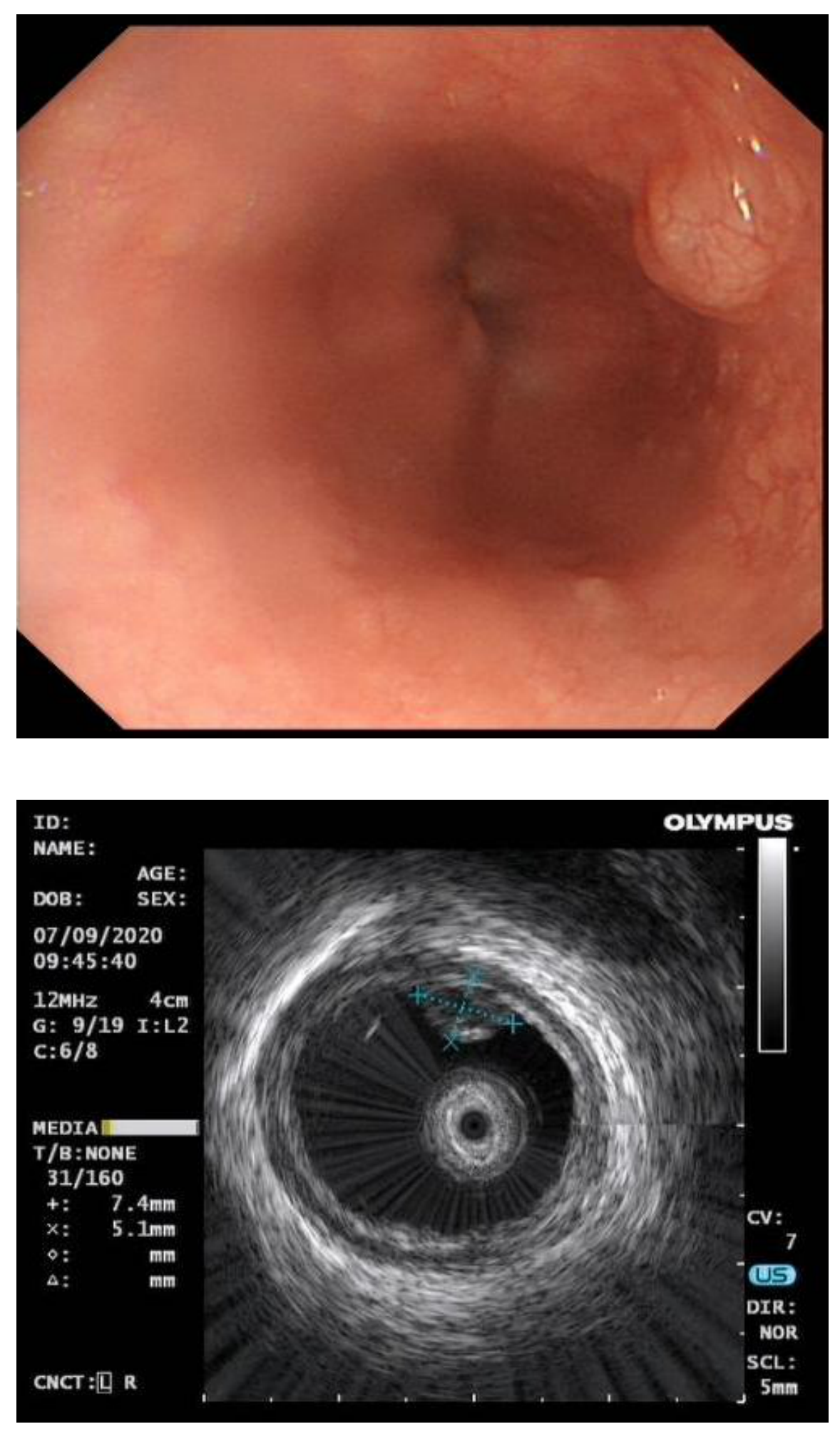

Fig1 Fig2 

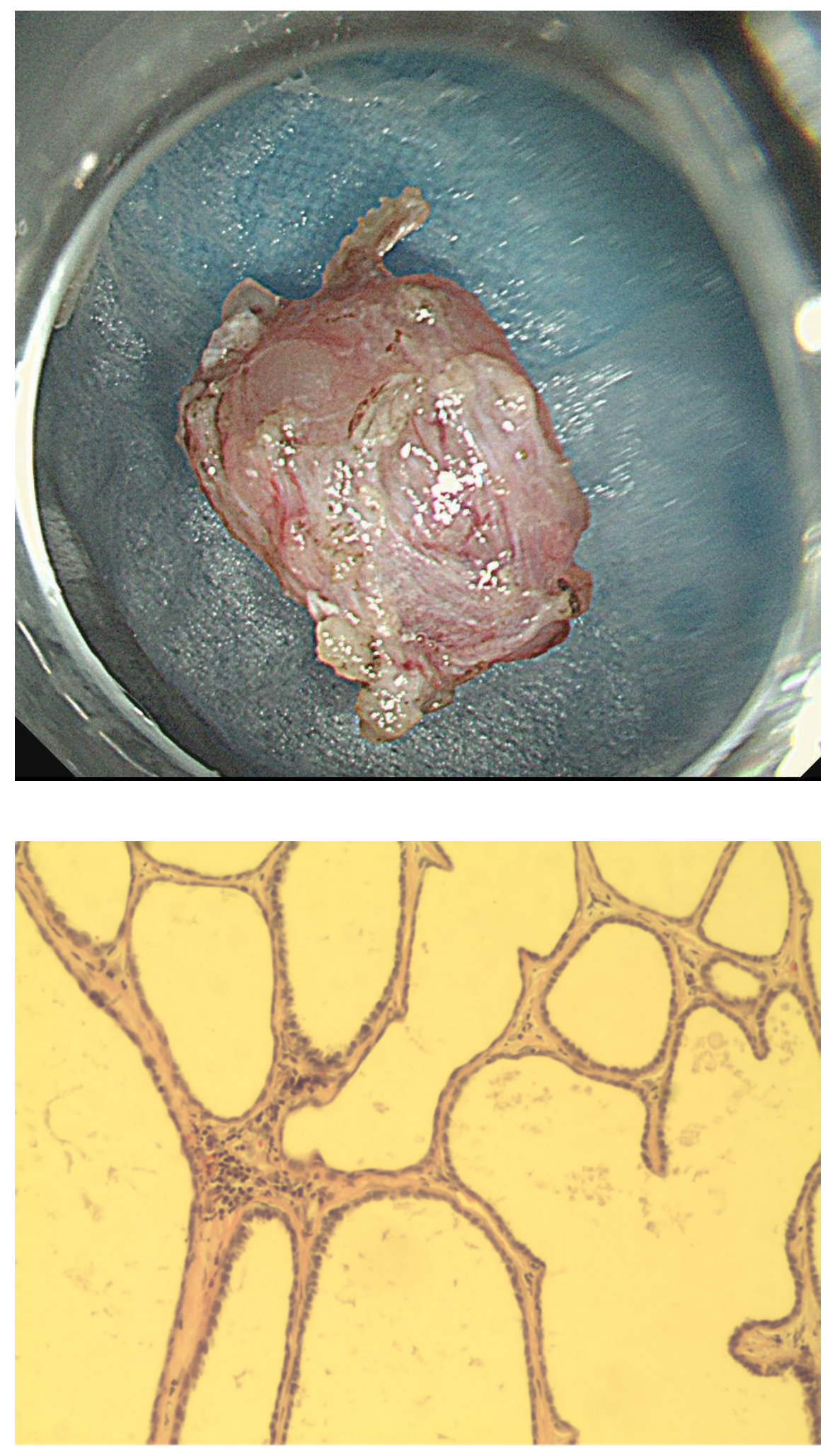

Fig3 Fig4 

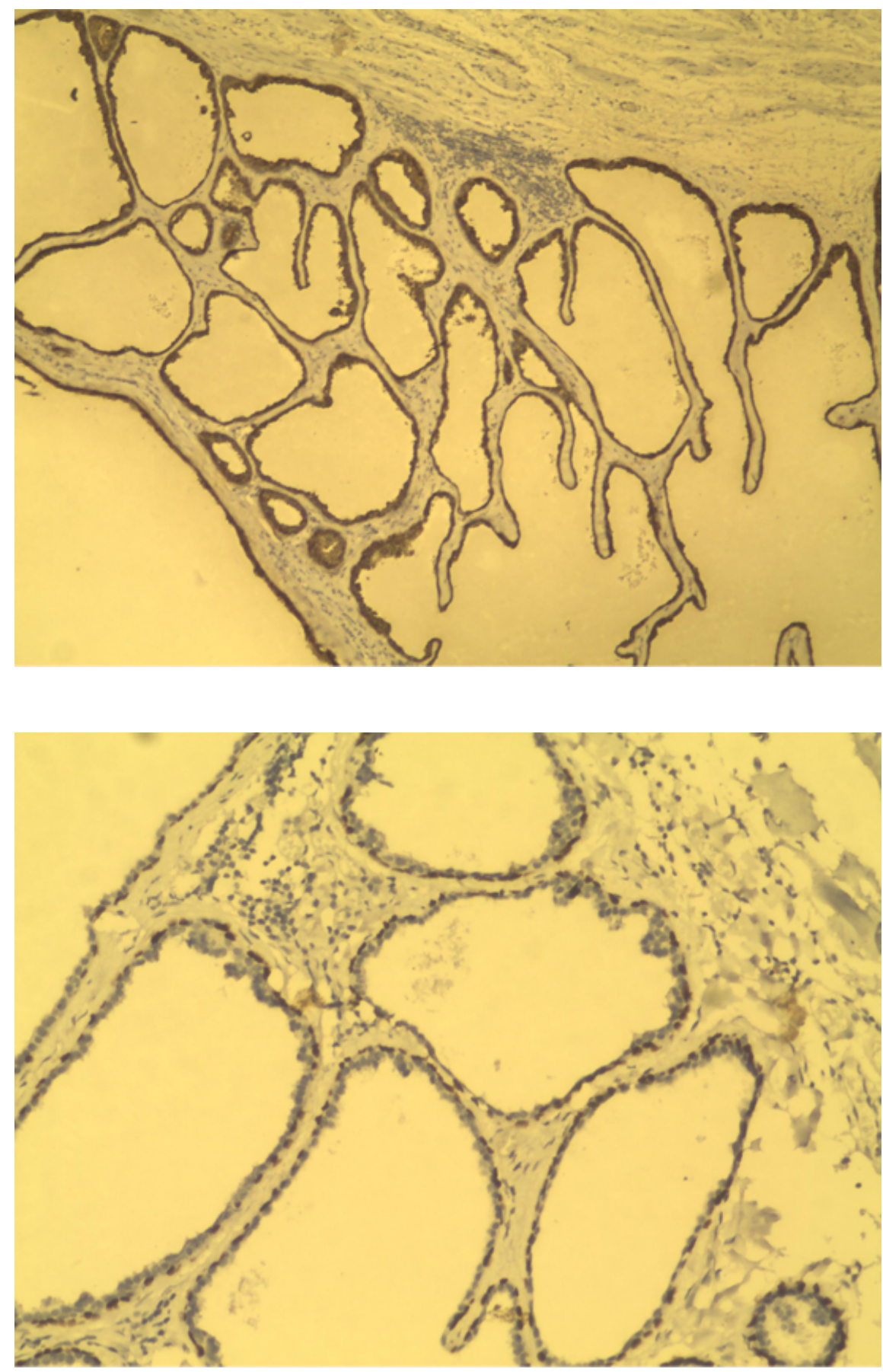

Fig5 Fig6

\section{Reference}

[1] Cannon D E,Szabo S,Flanary V A. Heterotopic Salivary tissue [J]. Am J Otolaryngol,2012,33(4):493-496.

[2]Hanke R,Comba I,Henriquez R,W Crespo M, Bhatia L. Salivary Gland Choristoma:A Rare Finding at the Gastroesophageal Junction[J]. Cureus,2020,12(2):e7138. 
[3]Abdul Karim L, Kwon DH, Ozdemirli M. Salivary gland heterotopia in the gastroesophageal junction:acase series and review of the literature[J].Case Rep Gastrointest Med,2018, 20 (1):e78581.

[4] Wang C, Chen L, Guo W, Zhu X, Liu Z, Salivary Gland Choriostoma in the Esophagus[J].Endoscopy, 2014, 46(11): 658-659.

[5]Schulberg SP,Serouya S,Cho M,Gadangi PK. Ectopic salivary gland found on rectal biopsy a rare pathological diagnosis[J].Int J Colorectal Dis,2020,35(5):967-969.

[6]Lee J H,Kim S,Kim S J. Heterotopic salivary gland tissue at the hepatic flexure of the large intestine:a case report[J].Korean J Gastroenterology,2018,72(4):213-216.

[7] Olajide T A,Agodirin S O,Ojewola.R W. Jejunal choristoma: a very rare cause of abdominal pain in children[J].Case Reports in Surgery,2014,2014:863647.

[8] Maffini F,Vingiani A,Lepanto D. Salivary gland choristoma in large bowel[J].Endoscopy,2012,44 (12) : $13-14$.

[9] Zhang Xia, Wang Lei. Endoscopic resection of submucosal salivary gland in gastric antrum: a case report[J]. Chinese community physician, 2012, 34303.

$[10] \mathrm{Su} \quad \mathrm{J} \quad \mathrm{M}, \mathrm{Hsu} \quad \mathrm{H} \quad \mathrm{K}, \mathrm{Hsu} \quad \mathrm{P} \quad$ I. Sialadenoma papilliferum of the esophagus[J].Am J Gastroenterol,1998,93(3):461-462.

[11] Takubo K,Esaki Y,Watanabe A. Adenoma accompanied by superficial squamous cell carcinoma of the esophagus[J]. Cancer,1993,71(8):2435-2438.

[12] Shibata M,Kusafuka K,Ono H. A rare submucosal tumor of the esophagus[J]. Gastroenterology, 2017,152 。

[13]Nie L, Wu HY ,Shen YH, Fan XS, Sun Q, Huang Q ,Chen J. Esophageal submucosal gland duct adenoma:a clinicopathological and immunohistochemical study with a review of the Literature[J]. Dis Esophagus,2016,29(8):1048-1053.

[14]Fujishima F,Nakamura Y,Kasajima A. Adenocarcinoma in the squamous lined esophagus without Barrett's mucosa,Probably arising from esophageal gland duct [J]. Esophagus,2015,12(3): 327-331.

[15]Haleem A,KFoury H,Al Juboury M. Paget's disease of the oesophagus associated with mucous gland carcinoma of the lower oesophagus [J]. Histopathology,2003,42(1):61-65.

[16]Shindo K, Bacon H E,Holmes E J. Ectopic gastric mucosa and gland ular tissue of a salivary type in the anal canal concomitant with a diverticu lumin hemorrhoidal tissue: report of a case[J]. Dis Colon Rectum,1972,15:57-62.

[17]Karaoglanoglu N, Eroglu A, Turylmaza. Oesophgel adenoid cystic carcinoma and its management options. Int J Clin Pract, 2005, 59: 1101-1103. 
White light endoscopic findings images of masses

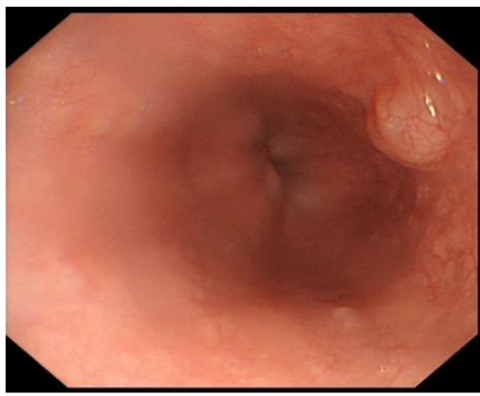

fig1. 
endoscopic ultrasound image of tumor

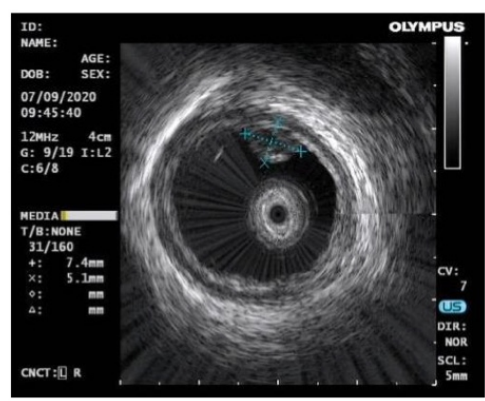

fig2 
Images of the endoscopic resection of the tumor

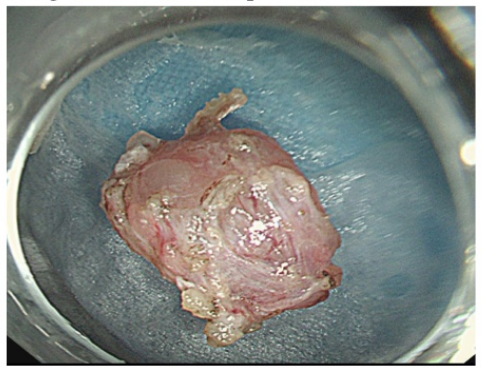

fig3 

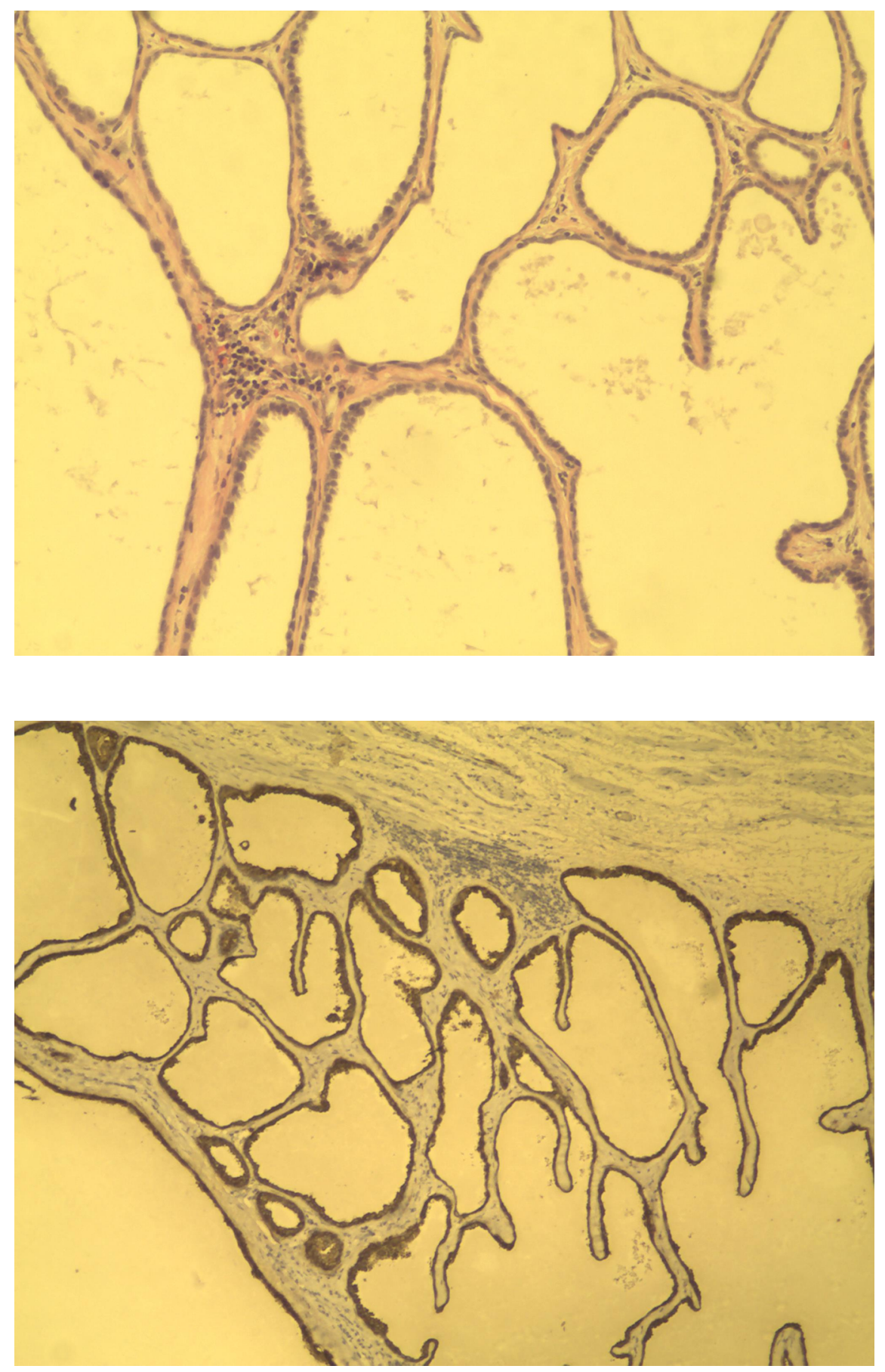


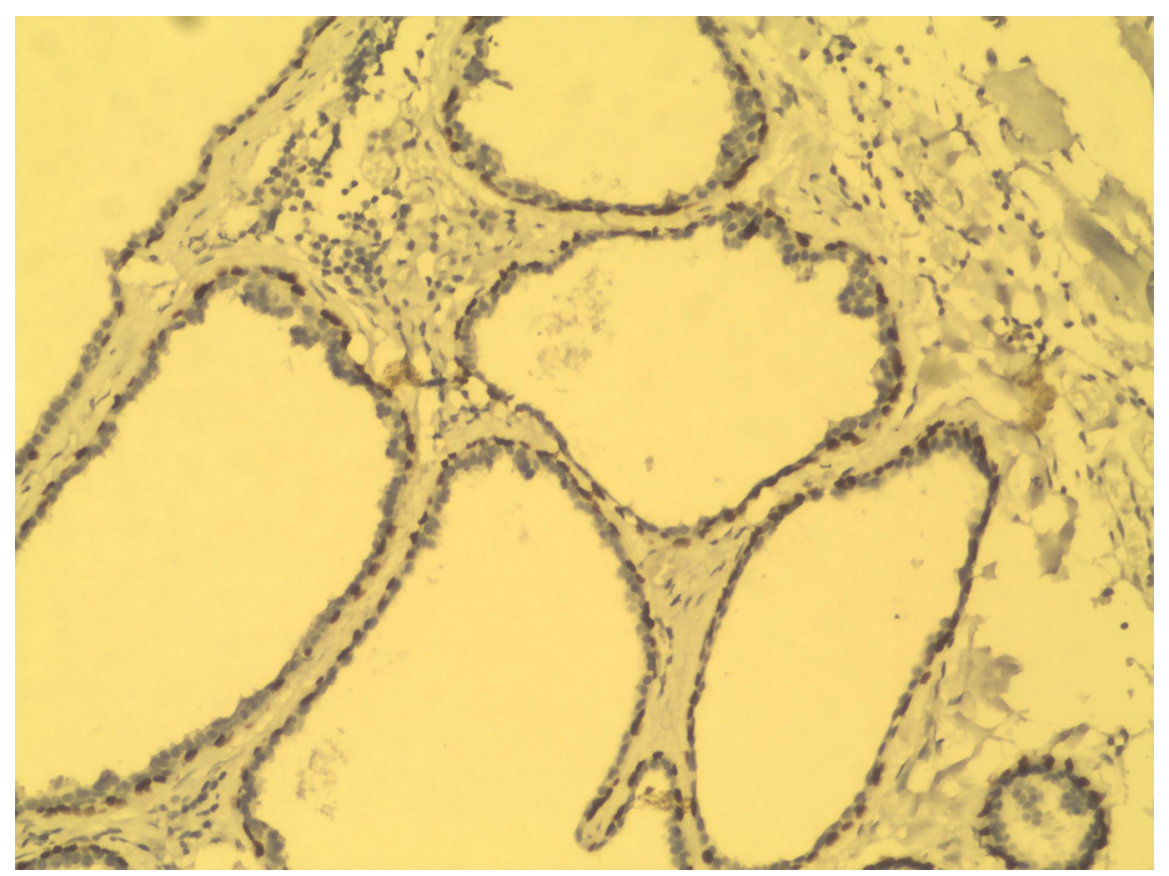

